\title{
TOLERANCIA A SUELOS ÁCIDOS EN POBLACIONES TROPICALES DE MAÍZ DEL CIMMYT1
}

\author{
H. Ceballos ${ }^{2}$, S. Pandey, E. B. Knapp, J. V. Duque ${ }^{3}$
}

\begin{abstract}
RESUMEN
Cinco poblaciones de maíz tropical del CIMMYT están siendo mejoradas para tolerancia a suelos ácidos mediante selección recurrente de hermanos completos. Se evaluaron tres ciclos de selección en un diseño de parcela dividida, con número variable de repeticiones, en cinco ambientes edáficos: uno con suelos normales (LNA) y los restantes cuatro con grados variables de estrés edáfico (LSA). Las ganancias genéticas observadas a través de las poblaciones fueron altamente significativas: $4,72 \%$ por ciclo, cuando los cinco ambientes fueron considerados, de $4,90 \%$ por ciclo cuando se consideraron únicamente las localidades LSA y de 4,21\% por ciclo para el ambiente LNA. Los resultados obtenidos sugie-ren buena variabilidad genética y alta heredabilidad de la tolerancia a los suelos ácidos. En ensayos comparativos de rendimiento, estas poblaciones rindieron un promedio de 2,29 y 6,25 t/ha en tres ambientes LSA y dos ambientes LNA, respectivamente. En estos mismos ambientes los testigos usados (Tuxpeño, Pool 26, y Suwan-1), rindieron un promedio de 1,28 y $5,56 \mathrm{t} / \mathrm{ha}$.
\end{abstract}

\begin{abstract}
Tropical corn populations tolerant to acid soils. Currently, five tropical maize populations from CIMMYT are being bred for tolerance to acid soils through a full-sib recurrent selection scheme. Three cycles of selection from each population were evaluated in a split-plot design, with a varied number of replications under five environments (with normal soils, and the remaining four with varying degrees of soil stress). The observed genetic gains from these populations were highly significant: $4.72 \%$ per cycle at all environments, $4.90 \%$ per cycle in acid soil environments (LSA), and $4.21 \%$ per cycle for the normal soil location (LNA). The results suggest adequate genetic variability and high heritability for tolerance to soil acidity. In comparative trials, these populations yielded an average of 2.29 and $6.25 \mathrm{t} / \mathrm{ha}$ in the three LSA and two LNA environments, respectively. Under the same environments, the controls used (Tuxpeño, Pool 26 and Suwan-1) yielded an average of 1.28 and $5.56 \mathrm{t} / \mathrm{ha}$.
\end{abstract}

\section{INTRODUCCION}

Aproximadamente $42 \%$ de todo el maíz cultivado, en países en vías de desarrollo, se encuentran en zonas tropicales (Edmeades et al., 1989). El rendimiento de estos maíces se ve altamente afectado por la baja fertilidad de los suelos, principalmente debido a los bajos contenidos de nitrógeno (Brewbaker, 1985), seguido por la deficiencia en fósforo $(\mathrm{P})$ el cual es frecuentemente inmobilizado por los altos contenidos de aluminio (Al) y hierro presentes en suelos ácidos. La gran mayoría de los suelos tropi- cales de América son ácidos (aproximadamente un 64\%) y su alta concentración de $\mathrm{Al}$ es, con frecuencia, la razón del fracaso del cultivo (Sánchez, 1977). Los suelos pueden ser ácidos bien sea porque sus materiales originales eran ácidos e inicialmente bajos en contenido de cationes o bien, porque estos elementos fueron removidos del suelo por los cultivos y/o el clima. Fertilizantes nitrogenados (sulfato de amonio, nitrato de amonio, urea, etc) también contribuyen a la acidificación de los suelos. Los suelos ácidos se caracterizan además de su frecuente toxicidad, por Al y manganeso, por deficiencias de calcio, magnesia,

\footnotetext{
1 Presentado en la XXXIX Reunión Anual del PCCMCA en Guatemala, América Central. 28 de marzo - 3 de abril, 1993.

2 Programa Suramericano del Centro Internacional de Mejoramiento de Maíz y Trigo (CIMMYT). Apdo. Aéreo 6713, Cali, Colombia.
} 
fósforo, hierro y molibdeno. Sin embargo, el máximo daño en los suelos ácidos de los trópicos ocurre por la toxicidad de Al, la cual interfiere con diversos procesos esenciales para el buen desarrollo radicular (Pandey et al., 1992).

La acidez del suelo puede ser corregida temporalmente mediante el encalado. Este procedimiento, sin embargo, es costoso y corrige sólo los estratos superiores del suelo (20-30 primeros $\mathrm{cm}$ ), y en muchos casos la fuente de cal se encuentra demasiado lejos, por lo que el costo de transporte lo hace económicamente impracticable. Es importante, por lo tanto, desarrollar materiales que presenten una buena tolerancia genética a la acidez del suelo y todos los problemas edáficos asociados con ella, ya que ésta es una solución permanente, barata y que eventualmente contribuye a una mejor conservación del ambiente. El Centro Internacional de Mejoramiento de Maíz y Trigo (CIMMYT) comenzó recientemente un programa para producir maíces tropicales con tolerancia a suelos ácidos. El presente estudio fue realizado para 1) estimar los progresos alcanzados luego de dos ciclos de selección recurrente en cuatro poblaciones tropicales de maíz y luego de cuatro ciclos de selección en una quinta población, y 2) comparar el potencial de rendimiento de estas poblaciones con otras poblaciones tropicales ampliamente difundidas, en condiciones de estrés edáfico y sin estrés.

\section{MATERIALES Y METODOS}

En 1985 se comenzó con el mejoramiento de la población SA3 (amarilla de ciclo completo) mediante selección recurrente de hermanos completos y evaluaciones de progenies en varias localidades. Cuatro ciclos de selección en SA3 se habían completado al iniciarse este estudio. En 1989 otras cuatro poblaciones fueron formadas a partir de materiales de diverso origen que mostraron buen comportamiento en evaluaciones en suelos ácidos. Dos de ellas son amarillas (SA4 y SA5) y dos son blancas (SA6 y SA7), siendo las poblaciones de un mismo color de grano, heteróticas entre sí. Estas cuatro poblaciones han sido mejoradas en forma similar a SA3 y ya se han completado dos ciclos de selección. Cada ciclo de selec- ción recurrente requirió un año calendario para ser completado: en el primer semestre de cada ciclo se evaluaban las familias de hermanos completos en tres o cinco ambientes de suelos ácidos (LSA) en la localidad de Carimagua, un ambiente de suelo ácido en Quilichao, y uno con suelos sin problemas de acidez (LNA) en Palmira, todos en Colombia (Cuadro 1). Evaluaciones se realizaban también en otros países tales como Brasil, Perú y Venezuela. Distintos tipos de estrés edáfico se lograban mediante el encalado de los suelos con cal dolomítica y la aplicación de superfostato triple. Durante el segundo semestre se recombinaban el mejor $10 \%$ de las progenies para regenerar las familias de hermanos completos e iniciar un nuevo ciclo de selección.

\section{Experimento 1.}

A comienzos de 1991, se hicieron aumentos de semilla de cada uno de los ciclos de selección de cada población ( $\mathrm{C} 0, \mathrm{Cl}$ y C2 de SA4 a SA7 y C0, C2 y C4 de SA3). La semilla fresca así obtenida se usó en evaluaciones a campo realizadas en cinco diferentes ambientes: Palmira (10 repeticiones), Quilichao 2 y 4 (total de 15 repeticiones), y Carimagua $\boldsymbol{E}$ y $\boldsymbol{C}$ (total de 19 repeticiones). Se utilizó un diseño de parcela dividida, arreglada en bloques al azar, donde la parcelas principales fueron las poblaciones y las subparcelas, los ciclos de selección dentro de cada población. El análisis estadístico se hizo usando PROC GLM (SAS,1985), para tener en cuenta la falta de balance debido al número variable de repeticiones en cada localidad. Poblaciones y ciclos de selección fueron considerados en el análisis estadístico como efectos fijos, y localidades como efectos aleatorios.

Las unidades experimentales se sembraron en surcos individuales de $5 \mathrm{~m}$ de largo, con 10 golpes por surco (separados $50 \mathrm{~cm}$ entre sí), con tres semillas por golpe y raleadas posteriormente a dos plantas por golpe. En el ensayo de Carimagua-C, cada unidad experimental contó con dos surcos. La cosecha se realizó a mano, desgranando sólo una porción de las mazorcas para medir la humedad del grano. En todos los casos se ajustó el rendimiento considerando un $80 \%$ de grano por mazorca y $15 \%$ de humedad. 
Cuadro 1. Localidades en Colombia donde las progenies de las poblaciones tolerantes a suelos ácidos son evaluadas cada año. Algunas poblaciones son evaluadas en localidades en otros países.

$\begin{array}{ccc}\text { Localidad } & \text { Saturación de Al } & \text { Concentración de P } \\ (\%) & (\text { ppm })\end{array}$

\begin{tabular}{lcc}
\hline Palmira $^{\text {ab }}$ & Sin toxicidad & Sin deficiencia \\
S. de Quilichao-1 $^{\text {ab }}$ & 45 & 6 \\
S. de Quilichao-2 $^{\text {ab }}$ & 65 & 12 \\
S. de Quilichao-4 $^{\text {b }}$ & 75 & 12 \\
Carimagua-A $^{\text {Carimagua-B }}$ & 75 & 12 \\
Carimagua-C $^{\mathrm{b}}$ & 45 & 12 \\
Carimagua-D & 45 & 6 \\
Carimagua-E & 75 & 6 \\
\end{tabular}

${ }^{a}$ Localidades donde se realizaron algunas de las evaluaciones del Experimento 2.

${ }^{\text {b}}$ Localidades donde se realizaron las evaluaciones de ciclos de selección del Experimento 1.

\section{Experimento 2.}

El último ciclo de selección de cada población fue comparado con tres materiales tropicales de amplia difusión (Tuxpeño Sequía, Pool 26 y Suwan-l) en tres ambientes LSA: Quilichao-1, Quilichao-2, y Sitiung (Indonesia, con $60 \%$ de saturación de Al) y dos ambientes LNA: Palmira y Suwan (esta última en Tailandia y la que dió, precisamente, el nombre a uno de los materiales usados como testigo). En cada localidad se sembraron seis repeticiones, en parcelas de surcos dobles de $5 \mathrm{~m}$ de largo. Además de las poblaciones tolerantes a suelos ácidos SA3, SA4, SA5, SA6 y SA 7, se incluyó una versión blanca (SA8) de la población SA3.

\section{RESULTADOS Y DISCUSION}

La germinación del CO de la población SA3 fue deficiente debido a problemas de insectos durante su almacenamiento. Este ciclo tuvo que ser aumentado nueva- mente y la semilla fresca así obtenida pudo ser usada en Quilichao-2. Las ganancias por selección en esta población, por lo tanto, están basadas únicamente en datos provenientes de esta localidad, y pueden estar subestimadas debido a que el $\mathrm{C} 0$ estuvo representado por semilla más fresca que los ciclos $\mathrm{C} 2$ y $\mathrm{C} 4$. No hubo diferencias importantes entre las dos localidades de Quilichao, ni tampoco entre las dos de Carimagua, por lo que los resultados se presentan considerando sólo tres ambientes Palmira, Carimagua y Quilichao.

En el Cuadro 2 se muestra en Análisis de Variancia para las variables Rendimiento, Días a Floración Femenina, y Prolificidad. Se efectuó una partición ortogonal de las sumas de cuadrados de los efectos principales, así como para aquellas interacciones que resultaron significativas para rendimiento. Se observaron diferencias significativas entre localidades: como era de esperarse, una gran porción de esta variabilidad se debió a diferencias entre la localidad no ácida (LNA) y los ambientes con suelos ácidos (LSA), así como diferencias entre Quilichao y 
Cuadro 2. ANOVA para ciclos de selección en cinco poblaciones evaluadas en cinco ambientes diferentes, en un diseño de parcela dividida, con número variable de replicaciones.

\begin{tabular}{|c|c|c|c|c|}
\hline Fuente de variación & $\begin{array}{l}\text { GL } \\
\text { t/ha }\end{array}$ & $\begin{array}{l}\text { Rendim. } \\
\text { Número de días }\end{array}$ & $\begin{array}{l}\text { Dîas a Flor Fem. } \\
\text { Maz./planta }\end{array}$ & Prolificidad \\
\hline LOCALIDADES (L) & 4 & $\mathbf{7 6 0 , 7 9} * *$ & $11589,02 * *$ & $\mathbf{7 , 0 2} * *$ \\
\hline Acidas (A) vs No Acidas (NA) & 1 & $2846,81 * *$ & $10442,32 * *$ & $25,17 * *$ \\
\hline Quilichao (Q) vs Carimagua (Cr) & 1 & $145,05 * *$ & $26139,33 * *$ & $1,01 * *$ \\
\hline Q1 V vs Q2 & 1 & 0,34 & $9774,37 * *$ & $1,23 * *$ \\
\hline $\mathrm{Cr} 1$ vs $\mathrm{Cr} 2$ & 1 & $50,95 * *$ & 0,06 & $0,67 *$ \\
\hline $\mathrm{REP}(\mathrm{R}) / \mathrm{L}$ & 39 & 3,25 & 58,42 & 0,11 \\
\hline POBLACIONES (P) & 4 & 3,43 & $106,66 * *$ & $\mathbf{0 , 5 8} *$ \\
\hline SA3 vs Resto & 1 & 9,34 & $358,46 * *$ & $1,87 * *$ \\
\hline SA4-SA5 vs SA 6-SA7 & 1 & 1,35 & 33,86 & 0,09 \\
\hline SA4 vs SA5 & 1 & 2,45 & 7,66 & 0,00 \\
\hline SA6 vs SA7 & 1 & 0,57 & 26,67 & 0,35 \\
\hline $\mathbf{P} * \mathbf{L}$ & 16 & $5,61 * *$ & 19,08 & $0,14 * *$ \\
\hline $\mathrm{P} * \mathrm{R} / \mathrm{L}$ & 154 & 1,42 & 15,54 & 0,05 \\
\hline CICLOS (C) & 2 & $13,11 * *$ & 49,61 & $0,21 *$ \\
\hline $\mathrm{C} 0$ vs $(\mathrm{C} 1-\mathrm{C} 2)$ & 1 & 2,06 & 2,69 & 0,03 \\
\hline $\mathrm{C} 1$ vs $\mathrm{C} 2$ & 1 & $24,18 * *$ & $96,53 *$ & $0,38 * *$ \\
\hline $\mathbf{C} * \mathbf{L}$ & 8 & 1,44 & $17,65 *$ & 0,03 \\
\hline $\mathrm{C} / \mathrm{P}$ & 7 & $3,04 *$ & 6,28 & 0,03 \\
\hline $\mathbf{C} / \mathbf{P} * \mathbf{L}$ & 28 & $1,11 *$ & 5,77 & 0,03 \\
\hline ERROR & 346 & 0,54 & 6,16 & 0,02 \\
\hline
\end{tabular}

", "* Significante a $\mathrm{P}=0,05$ y $\mathrm{P}=0,01$, respectivamente

Carimagua. Los rendimientos promedio de los dos ensayos sembrados en Carimagua fueron estadísticamente significativos, pero no en los ensayos de Quilichao.

Los promedios, poblacionales no fueron diferentes cuando los cinco ambientes fueron tenidos en cuenta, pero cuando se realizaron los análisis sólo para LNA o LSA, entonces sí las diferencias alcanzaron significancia estadística para las tres variables analizadas (Cuadro 3). La interacción población * localidad fue significativa para rendimiento y Prolificidad, siendo una gran porción de esta variabilidad debida al componente LNA vs LSA * SA3 vs Resto. En efecto, la población SA3 se destaca en LSA pero fue la que menos rindió en Palmira (LNA). Otro componente importante de esta interacción fue la de LNA vs LSA * SA6 vs SA 7, ya que la población SA6 fue la de más alto rendimiento en Palmira pero la más pobre através de LSA, mientras que SA7 tuvo un comportamiento pobre en Palmira pero mejor que SA6 en Carimagua (Cuadro 3).

Los promedios de los ciclos através de poblaciones fueron estimados con la exclusión de la población SA3, debido a la baja germinación de SA3-C0 y a que los ciclos evaluados de esta población eran diferentes a aquéllos de las restantes poblaciones. Se observaron diferencias significativas entre las medias de ciclos para rendimiento y Prolificidad. Gran parte de estas diferencias se deben a cambios entre $\mathrm{C} 1$ y C2. Através de las poblaciones y teniendo en cuenta todas las localidades, o bien LSA, el C0 superó al C1, aunque las diferencias no fueron significativas. Por otra parte, el C2 superó a C0 y CIen todos los ambientes (Cuadro 3). 
Cuadro 3. Promedios de tres ciclos de selección de cinco poblaciones evaluadas en cinco ambientes*.

\begin{tabular}{|c|c|c|c|c|c|c|}
\hline \multirow[t]{2}{*}{ Pob. } & \multirow[t]{2}{*}{ Ciclo } & \multicolumn{5}{|c|}{ Através de 5 ambientes Suelos ácidos Palmira } \\
\hline & & $\begin{array}{c}\text { Rendim. } \\
\text { t /ha }\end{array}$ & $\begin{array}{l}\text { Días a } \\
\text { flor. }\end{array}$ & $\begin{array}{l}\text { Número } \\
\text { maz./pl. }\end{array}$ & $\begin{array}{c}\text { Rendim. } \\
\text { t/ha }\end{array}$ & $\begin{array}{c}\text { Rendim. } \\
\text { t/ha }\end{array}$ \\
\hline $\mathrm{SA} 3$ & $\mathrm{C} 0$ &,-- &,-- &,-- &,-- & \\
\hline SA3 & $\mathrm{C} 2$ & 2,90 & 71,0 & 0,87 & 2,40 & 4,60 \\
\hline SA3 & $\mathrm{C} 4$ & 4,09 & 70,3 & 0,92 & 3,05 & 7,65 \\
\hline \multicolumn{2}{|c|}{ Media de SA3 } & 3,36 & 70,7 & 0,92 & 2,64 & 6,12 \\
\hline SA4 & $\mathrm{CO}$ & 3,23 & 71,3 & 0,76 & 2,02 & 7,25 \\
\hline SA4 & $\mathrm{Cl}$ & 3,43 & 72,4 & 0,77 & 2,14 & 7,70 \\
\hline SA4 & $\mathrm{C} 2$ & 3,43 & 71,1 & 0,76 & 2,07 & 7,78 \\
\hline \multicolumn{2}{|c|}{ Media de SA4 } & 3,36 & 71,6 & 0,76 & 2,07 & 7,58 \\
\hline SA5 & $\mathrm{CO}$ & 3,26 & 72,2 & 0,76 & 1,94 & 7,61 \\
\hline SA5 & $\mathrm{C} 1$ & 2,91 & 73,0 & 0,72 & 1,71 & 6,86 \\
\hline SA5 & $\mathrm{C} 2$ & 3,30 & 71,5 & 0,80 & 2,07 & 7,34 \\
\hline \multicolumn{2}{|c|}{ Media de SA5 } & 3,16 & 72,2 & 0,76 & 1,91 & 7,27 \\
\hline SA6 & $\mathrm{C} 0$ & 3,01 & 72,6 & 0,69 & 1,75 & 7,30 \\
\hline SA6 & $\mathrm{C} 1$ & 3,05 & 73,2 & 0,66 & 1,65 & 7,82 \\
\hline SA6 & $\mathrm{C} 2$ & 3,36 & 72,8 & 0,72 & 1,99 & 8,02 \\
\hline \multicolumn{2}{|c|}{ Media de SA6 } & 3,14 & 72,9 & 0,69 & 1,80 & 7,72 \\
\hline SA7 & $\mathrm{CO}$ & 2,85 & 73,1 & 0,73 & 1,85 & 6,24 \\
\hline SA7 & $\mathrm{C} 1$ & 2,91 & 73,1 & 0,73 & 1,79 & 6,70 \\
\hline SA7 & $\mathrm{C} 2$ & 3,39 & 72,1 & 0,73 & 2,14 & 7,64 \\
\hline \multicolumn{2}{|c|}{ Media de SA7 } & 3,04 & 72,8 & 0,76 & 1,93 & 6,86 \\
\hline \multirow{3}{*}{\multicolumn{2}{|c|}{$\begin{array}{l}\text { Media de } \mathrm{C} 0 \\
\text { Media de } \mathrm{C} 1 \\
\text { Media de } \mathbf{C 2}\end{array}$}} & 3,09 & 72,3 & 0,74 & 1,89 & 7,10 \\
\hline & & 3,08 & 72,9 & 0,72 & 1,82 & 7,27 \\
\hline & & 3,37 & 71,9 & 0,75 & 2,07 & $\mathbf{7 , 7 0}$ \\
\hline \multirow{3}{*}{\multicolumn{2}{|c|}{$\begin{array}{l}\text { DMS-1 } \\
\text { DMS-2 } \\
\text { DMS3 }\end{array}$}} & 0,75 & 1,32 & 0,07 & 0,37 & 0,89 \\
\hline & & 0,28 & 0,85 & 0,04 & 0,16 & 0,42 \\
\hline & & 0,65 & 1,38 & 0,10 & 0,44 & 0,65 \\
\hline
\end{tabular}

- Cuatro localidades con suelos ácidos: Carimagua-E (55\% Sat. Al.- 10 ppm P), Carimagua-C (45\% Sat. Al - 6 ppm P), Quilichao2 (65\% Sat. Al- 10 ppm P), Quilichao-4 (75\% Sat, Al - 12 ppm P), y Palmira (suelos normales).-Promedios de ciclos através de poblaciones con la exclusión de la población SA3.

* Diferencias mínimas significativas $(\mathrm{p}=0,05)$ para comparaciones entre ciclos dentro de poblaciones (DMS-1), entre ciclos através de poblaciones (DMS-2), y entre poblaciones através de ciclos (LSD-3). 
Cuando las ganancias se analizaron através de poblaciones (Cuadro 4), éstas fueron altamente significativas $(\mathrm{P}=0,01)$ através de los cinco ambientes $(146 \mathrm{~kg} / \mathrm{ha} / \mathrm{ci}$ clo), através de las LSA ( $93 \mathrm{~kg} / \mathrm{ha} /$ ciclo), en Quilichao (146 kg/ha/ciclo), en Palmira (299 kg/ha/ciclo), y significativa $(\mathrm{P}=0,05)$ através de los dos ensayos de Carimagua (94 kg/ha/ciclo), Ganancias en poblaciones individuales fueron significativas para SA6 en Carimagua $(234 \mathrm{~kg} / \mathrm{ha}-$ /ciclo), y SA7 en Quilichao (191 kg/ha/ciclo) y Palmira (703 kg/ha/ciclo).

El Cuadro 5 muestra los rendimientos (Experimento 2) de los materiales evaluados en las localidades individuales, através de las localidades LSA y através de las localidades LNA. Las poblaciones SA3 y SA8 fueron las de mayor rendimiento através de las localidades LSA con 2,82 y 2,76 t/ha, respectivamente. En los ambientes LNA, todas las poblaciones tolerantes superaron las $6 \mathrm{t} / \mathrm{ha}$ de rendimiento (promedio 6,25 t/ha), mientras que Tuxpeño Sequía, Pool26 y Suwan-1, rindieron por debajo de este valor (promedio 5,56 t/ha), Estos resultados demuestran claramente que las poblaciones tolerantes a suelos ácidos no sólo tienen un excelente potencial de rendimiento en estos suelos con problemas de toxicidad de $\mathrm{Al}$ y deficiencia de $\mathrm{P}$, sino que también son altamente competitivas en ambientes sin problemas edáficos y de buena productividad.
El mejoramiento de la tolerancia a suelos ácidos se ve afectado por numerosos factores, principalmente: i) las evaluaciones a campo, bajo condiciones de suelos ácidos, se caracterizan usualmente por grandes sumas de cuadrados residuales debidas a la variabilidad ocasionada por la falta de uniformidad del suelo, ii) existen numerosos factores que determinan la pobre fertilidad de los suelos ácidos (bajo $\mathrm{pH}$, deficiencia de $\mathrm{P}$, deficiencia de $\mathrm{Ca}$, toxicidad del $\mathrm{Al}$, toxicidad de $\mathrm{Mn}$, etc). Si la tolerancia genética a cada uno de estos factores es independiente (una suposición razonable mientras no se pruebe lo contrario), es de esperarse que el mejoramiento simultáneo para todos los factores sea, en el mejor de los casos, lento. A pesar de estos inconvenientes, se ha podido observar que se pueden realizar ganancias importantes y rápidas en la tolerancia del maíz a los suelos ácidos através de ambientes ampliamente diversos.

Si bien el progreso en la población SA3 (6,37\% ó 121 $\mathrm{kg} / \mathrm{ha} / \mathrm{ciclo}$ ) no fue significativo y sólo pudo evaluarse satisfactoriamente en Quilichao-2, resultados de un estudio diferente mostraron ganancias de ] 3,9\% por ciclo (para ciclos C0, C1 y C2), luego de evaluaciones bajo 6 LSA (Granados et al. 1993). Ya se mencionó anteriormente que el progreso en SA3 puede haber sido subestimado ya que C0 contaba con semilla más fresca que $\mathrm{C} 2$ y C4. Las

Cuadro 4. Ganancias obtenidad por selección para tolerancia a suelos ácidos en 5 poblaciones evaluadas en cinco ambientes con grados variables de estrés edáfico.

\begin{tabular}{|c|c|c|c|c|c|}
\hline \multicolumn{5}{|c|}{ Población } & \multirow[t]{3}{*}{ Ganancias } \\
\hline \multirow[t]{2}{*}{ Todos los ambientes } & \multicolumn{3}{|c|}{ Localidades con suelos ácidos } & \multirow[b]{2}{*}{ Palmira } & \\
\hline & Todas & Carimagua & Quilichao & & \\
\hline SA3 &,-- &,-- &,-- & 6,37 &,-- \\
\hline $\mathrm{SA} 4$ & 2,36 & 0,79 & $-0,13$ & 3,11 & 3,63 \\
\hline SA5 & 0,38 & 2,95 & 0,55 & 9,00 & $-1,72$ \\
\hline SA6 & 6,81 & 7,64 & $10,50^{*}$ & $-2,61$ & 4,93 \\
\hline SA7 & $10,01 *$ & 8,56 & 5,16 & $18,02 *$ & $11,26 * *$ \\
\hline Através de Pobs, & $4,72 * *$ & $4,90 * *$ & $3,86^{*}$ & 11,31 & $4,21 * *$ \\
\hline
\end{tabular}

Ganancias estimadas como \% del coeficiente de regresión sobre el rendimiento observado para el respectivo C0.

* Ganancias para la población SA3 basada únicamente en datos de Quilichao-2.

1 Ganancias através de poblaciones estimadas con la exclusión de la población SA3, excepto para Quilichao. 
Cuadro 5. Comparación de los rendimientos del último ciclo de selección con tres testigos de amplia difusión en tres ambientes con suelos ácidos y dos con suelos sin problemas edáficos $\$$.

\begin{tabular}{|c|c|c|c|c|c|c|c|}
\hline \multirow[b]{3}{*}{ Población } & \multicolumn{6}{|c|}{$R$ endimiento ( $t / h a)$} & \\
\hline & \multicolumn{3}{|c|}{ Suelos no ácidos } & \multicolumn{4}{|c|}{$\begin{array}{l}\text { Suelos ácidos } \\
\end{array}$} \\
\hline & Palmira & Suwan & Promedio & Sitiung & Lote-1 & Lote-2 & Promedio \\
\hline $\mathrm{SA} 3-\mathrm{C} 4$ & 8,29 & 4,98 & 6,30 & 2,26 & 3,35 & 2,85 & 2,82 \\
\hline $\mathrm{SA} 4-\mathrm{C} 2$ & 8,15 & 4,57 & 6,00 & 2,83 & 2,46 & 1,84 & 2,38 \\
\hline SA5-C2 & 8,14 & 5,01 & 6,26 & 2,89 & 2,76 & 2,09 & 2,58 \\
\hline SA6-C2 & 8,71 & 4,80 & 6,37 & 2,29 & 1,73 & 1,04 & 1,68 \\
\hline $\mathrm{SA} 7-\mathrm{C} 2$ & 8,68 & 4,76 & 6,33 & 2,79 & 2,19 & 0,95 & 1,97 \\
\hline $\mathrm{SA} 8-\mathrm{C} 0$ & 8,35 & 4,70 & 6,16 & 2,83 & 3,38 & 2,07 & 2,76 \\
\hline Tuuxpeño & 7,68 & 4,54 & 5,79 & 2,16 & 0,88 & 0,19 & 1,08 \\
\hline Pool26 & 7,73 & 4,37 & 5,71 & 1,85 & 1,19 & 0,40 & 1,15 \\
\hline Suwan-1 & 7,00 & 3,95 & 5,17 & 2,33 & 1,43 & 1,07 & 1,61 \\
\hline Coef. Var. & 6,14 & 12,80 & 9,26 & 19,76 & 29,18 & 43,98 & 26,03 \\
\hline DMS $(5 \%)$ & 0,71 & 0,67 & 0,49 & 0,56 & 0,73 & 0,72 & 0,74 \\
\hline
\end{tabular}

Lotes 1 y 2 (S. de Quilichao) y Palmira localizados en Colombia, Suwan en Tailandia y Sitiung en Indonesia.

ganancias en las poblaciones blancas SA6 y SA7 fueron, en general, mayores que las observadas en las poblaciones amarillas SA4 y SA5. La composición gen ética de estas poblaciones es diferente, asícomo los niveles originales de tolerancia en sus respectivos $\mathrm{C} 0$ s. Estas diferencias podrían explicar la respuesta diferencial a la selección en estas poblaciones. Las ganancias en Quilichao fueron más altas que las observadas en Carimagua. En parte, esto podría deberse a la falta de fertilidad crónica de los suelos de Carimagua (baja materia orgánica y deficiencia no corregida de K). Esta muy baja fertilidad (no necesariamente característica de la condición ácida de estos suelos), podría haber enmascarado los verdaderos progresos logrados para la acidez de estos suelos. La falta de significancia para las ganancias dentro de poblaciones puede explicarse por el reducido número de ciclos evaluados en cada población (sólo tres) y a la magnitud del error experimental típica en este tipo de evaluaciones.
Es interesante destacar que se pudo observar progresos, en una dada población, para diversos ambientes en forma simultánea. Tal es el caso de la población SA7 con ganancias de 5,16.; 18,02**, y 11,26**, para Carimagua, Quilichao y Palmira, respectivamente. Sólo en dos casos (SA6 en Quilichao y SA5 en Palmira) se observaron ganancias negativas relativamente importantes, aunque no significativas. Estos resultados demuestran que es posible lograr ganancias genéticas importantes para la tolerancia a suelos ácidos en forma relativamente rápida, y sugieren que la heredabilidad para este carácter es relativamente alta, coincidiendo con resultados obtenidos por otros autores (Magnavaca et al. 1987, Duque Vargas et al., 1994).

En general se pudo observar un retroceso entre $\mathrm{C} 0 \mathrm{y}$ Cl. En parte, esto puede deberse a la forma en que, hasta el C1, estas poblaciones fueron mejoradas. En efecto, parte importante de la selección se basaba en calificaciones 
visuales para el vigor de las familias durante el desarrollo (en aquellas condiciones en que el estrés edáfico era muy severo, ésta era la única información usada ya que los rendimientos eran demasiado bajos para podcr ser satisfactoriamente pesados en el campo). Observaciones posteriores (datos no presentados) demostraron que el vigor de las familias durante el desarrollo tiene una correlación muy baja con el rendimiento. En algunas familias, un mejor vigor se lograba a expensas de un rendimiento de grano nulo. A partir del C2, se dejó de usar el vigor como un carácter a tencr en cuenta en ]a selección, la que pasó a basarse principalmente en el rendimiento, fecha de floración femenina, y proJificidad.

Los resultados del Experimento 2 coinciden con los del Experimento $1 \mathrm{y}$ resaltan el potencial de rendimiento de estos materiales no sólo en suelos ácidos sino también en aquéllos sin problemas edáficos y alta productividad.

\section{CONCLUSIONES}

1. Las ganancias observadas sugieren que existe suficiente variabilidad genética para la tolerancia a los suelos ácidos en las poblaciones evaluadas y que el método de mejoramiento empleado es adecuado.

2. Un aumento en la tolerancia a suelos ácidos no disminuye el potencial de rendimiento en sucios normales.

3. Las poblaciones mejoradas mostraron un excelente potencial de rendimiento tanto en suelos ácidos como en suelos productivos.

\section{DEDICATORIA}

Dedicamos este artículo a nuestro colega Javier Duque Vargas, recientemente fallecido.

\section{LITERATURA CITADA}

BREWBAKER, J. L. 1985. The tropical environment for maize cultivation. En: Breeding strategies for maize production improvement in the tropics. A. Brandolini y F. Salamini Eds. FAO. Roma, Italia.

DUQUE VARGAS, 1; PANDEY, S.; GRANADOS, G.; CEBALLOS, H.; KNAPP, E. 1994. Inheritance of tolerance to soil acidity in tropical maize. Crop. Sci. 34 (En impresión) .

EDMEADES, G. O.; BOLAÑOS, J.; LAFFITTE, H. R.; RAJARAN, S. ; PFEIFFER, W.; FISCHER, R. A. 1989. Traditional approaches to breeding for drought resistance in cereals. En: F.W.G. Baker (Ed.) Drought resistance in cereals - Theory and Practice, ICSU Press, Paris, Francia.

GRANADOS, G.; PANDEY, S.; CEBALLOS, H. Response to selection for tolerance to acid soils in a tropical maize population. Crop. Sci. 33: 936-940

PANDEY, S.; CEBALLOS, H.; KNAPP, E. B.; V ARGAS, J. D. 1992. Genetic variability in maize for adaptation to acid soils. Simposio Internacional sobre Estresse ambiental: O milho em perspectiva. Marzo 8-13, 1992, Belo Horizonte (Brasil).

MAGNAVACA, R.; GARDNER, C.O.; CLARK,R. B. 1987. Inheritance to aluminum tolerance in maize. En: W.H. Gabelman y B.C. Loughman (Eds), Genetic aspects of plant mineral nutrition. Martinus Nijhoff. La Haya, Holanda.

PANDEY, S.;CEBALLOS, H.; KNAPP, E. B.; V ARGAS, J. D. 1992. Genetic variability in maize for adaptation to acid soils. En: Simposio Internacional sobre Estress ambiental: O milho en perspectiva. Marzo 8-13, 1992. Belo Horizonte, Brasil.

SAS; USER'S GUIDE: STATISTICS. 1985. Versión 5 Ed. SAS Institute, Inc., Cary, N.e., USA.

SANCHEZ, P. A. 1977. Advances in management of Oxisols and Ultisols in tropical South America. En: International Seminar on soil, environment, and fertility management in intensive agriculture. Tokyo, Japón. 